\title{
Insect bites as a trigger factor of eosinophilic cellulitis
}

\author{
Monika Kucharczyk ${ }^{1, A, C-D}$, Malgorzata Slowik-Rylska ${ }^{1,2, A-B}$, Beata Krecisz ${ }^{1,2, A, E-F}$ \\ ${ }^{1}$ Faculty of Medicine, Jan Kochanowski University, Kielce, Poland \\ 2 Dermatology Department, Provincial General Hospital, Kielce, Poland \\ A - Research concept and design, B - Collection and/or assembly of data, C - Data analysis and interpretation, \\ $D$ - Writing the article, E-Critical revision of the article, F- Final approval of article
}

Kucharczyk M, Slowik-Rylska M, Krecisz B. Insect bites as a trigger factor of eosinophilic cellulitis. Ann Agric Environ Med. 2019; 26(2): 256-259. doi: $10.26444 /$ aaem/97367

\begin{abstract}
I Abstract
Introduction. The study presents the case report of a Wells Syndrome in a 18-year -Id female. Wells Syndrome is a rare inflammatory skin disorder which seems to present an abnormal eosinophilic response to a number of triggers.

Objective. The aim of the study is to discuss several problems related to the diagnosis and treatment of Wells Syndrome. Materials and method. Medical examination, blood tests, abdominal ultrasound and skin biopsy were performed.

Results. Medical examination revealed plaques with tense blisters on feet and erythematous lesions on trunk. Blood tests showed raised anti-streptolysin O level. Skin biopsy revealed features of eosinophilic cellulitis. Oral administration of $0.5 \mathrm{mg}$ per day prednisolone and antihistaminics with local therapy resulted in good clinical response.

Conclusions. Sometimes several biopsy are required to establish diagnosis. In some cases lower doses of corticosteroids with antihistaminics and local anti-inflammatory treatment can by sufficient in treatment.
\end{abstract}

\section{Key words}

eosinophilic cellulitis, Wells Syndrome, flame figures, morphea-like lesions

\section{CASE REPORT}

A previously healthy 18 -year-old female presented to the Dermatology Department complaining about the sudden appearance of erythematous, infiltrated plaques, with associated tense blisters filled with serous fluid on the dorsal and posterior aspect of both feet (Fig. 1). The lesions were pruritic, and the patient was afebrile. Physical examination revealed a $5 \times 5 \mathrm{~cm}$ erythematous, tender plaque located on the right side of the trunk, moderately itchy (Fig. 2). The patient reported that the lesions appeared a few days before her presentation, after she had been walking in a meadow. She denied a history ingestion of medications or other intercurrent illness. The localization, morphology of cutaneous lesions and history of spending a large part of her time outdoors during the summer months prior to the development of first symptoms, suggested an arthropod assault. The patient identified the suspected arthropod as a mosquito.

Physical examination and blood tests were performed, including: anti-streptolysin O, ANA, Ig E, CRP, ERS, morphology, renal and liver function tests, urinalysis, Borrelia burgdoferi antibodies and stool testing for ova and parasites were taken. Additionally, two skin biopsy specimens were taken from the feet for histopathology, and direct immunofluorescence examinations were performed. An abdominal ultrasound examination was conducted.

Results of laboratory examinations during first hospitalization. Blood tests demonstrated raised antistreptolysin O $598 \mathrm{IU} / \mathrm{ml}$ (0-200), ANA 1:320 homogeneousgranular pattern, Extractable Nuclear Antigen Antibodies

Address for correspondence: Monika Kucharczyk, Faculty of Medicine, Jan Kochanowski University, Kielce, Poland

e-mail: kucharczykmonik@gmail.com

Received: 27.08.2018; accepted: 08.10.2018; first published: 30.10.2018

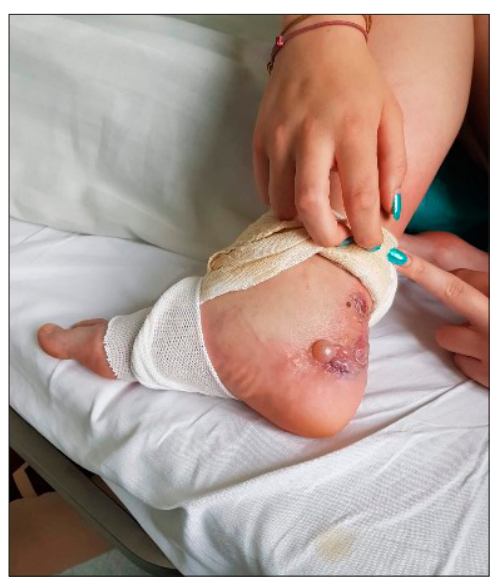

Figure 1. Erythematous, infiltrated plaques with tense blisters filled with serous fluid on foot

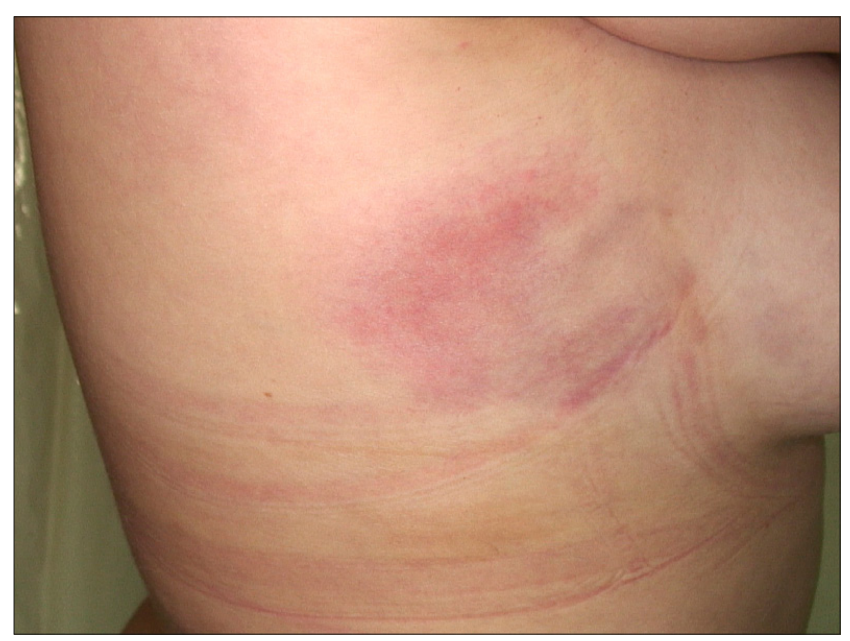

Figure 2. Erythematous, tender plaque located on right side of the trunk 
(ENA) Panel and dsDNA antibodies were negative. Laboratory investigations included: Ig E, CRP, ERS, morphology, renal and liver function tests, and urinalysis were unremarkable. Abdominal ultrasound examination was normal. Borrelia burgdoferi Ig M, Ig G antibodies and stool testing for ova and parasites were negative. Histopathology examination revealed arthropod bite reaction and direct immunofluorescence findings were not specific.

Diagnosis: The patient was diagnosed with arthropod bite reaction.

Treatment: A short course of oral corticosteroids was administrated with initial good clinical response. Eruptions resolved within 10 days.

Follow-up: Relapse of bullous lesions on the feet and appearance of new annular infiltrated morphea-like lesions, with central grey-blue discolouration and violaceous border, measuring $20 \times 15 \mathrm{~cm}$ on left side of the trunk was observed in about one month (Figs. 3, 4). However, it cannot be excluded that the recurrence of the disease was caused by another exposition to insect bites.

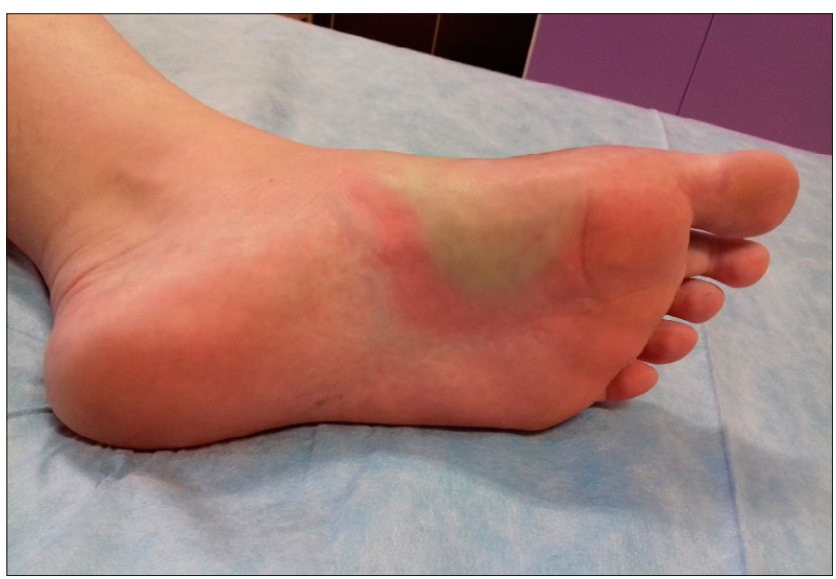

Figure 3. Urticaria-like lesions on feet

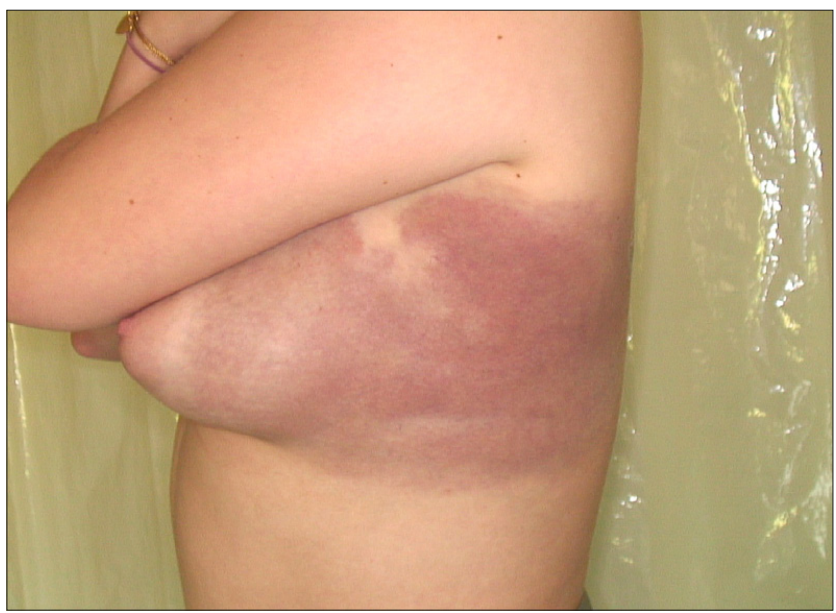

Figure 4. Morphea-like lesions, with central grey-blue discoloration and violaceous border on left side of trunk

The diagnostic process was extended - additional skin biopsy from an erythematous-edematous area on back, further laboratory studies including reumathoid factor and tumour markers, such as cancer antigen 125 (Ca 125), carcinoembryonic antigen (CEA), and Alpha-fetoprotein (AFP) tests, Anticardiolipin Antibodies (ACA) IgG, IgM and Anti-neutrophil cytoplasmic antibodies (pANCA, cANCA) were performed.

Results of laboratory examinations during second hospitalization. Histopathology assessment showed dermal eosinophils and flame figures (Figs. 5, 6). Other laboratory studies were without any abnormalities.

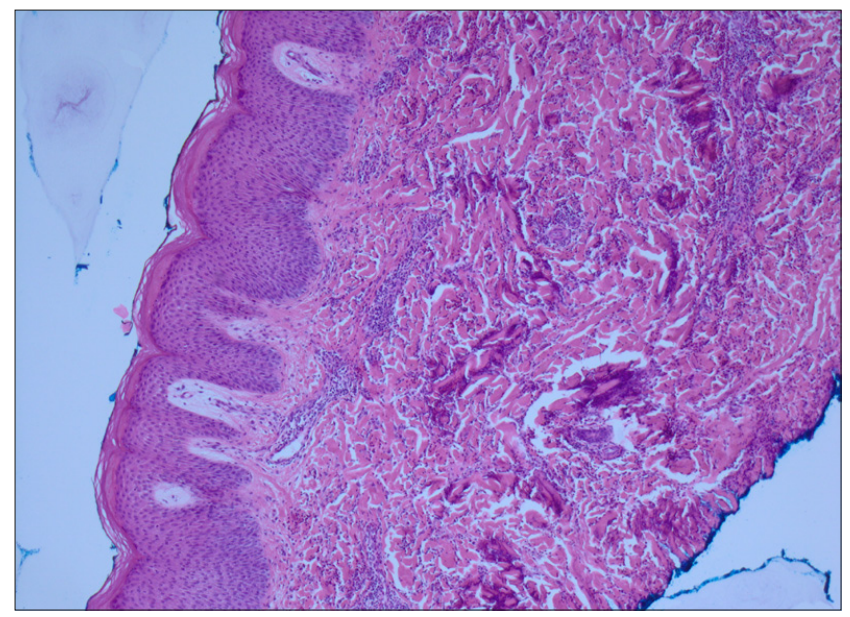

Figure 5. Eosinophilic infiltration of the dermis and flame figures

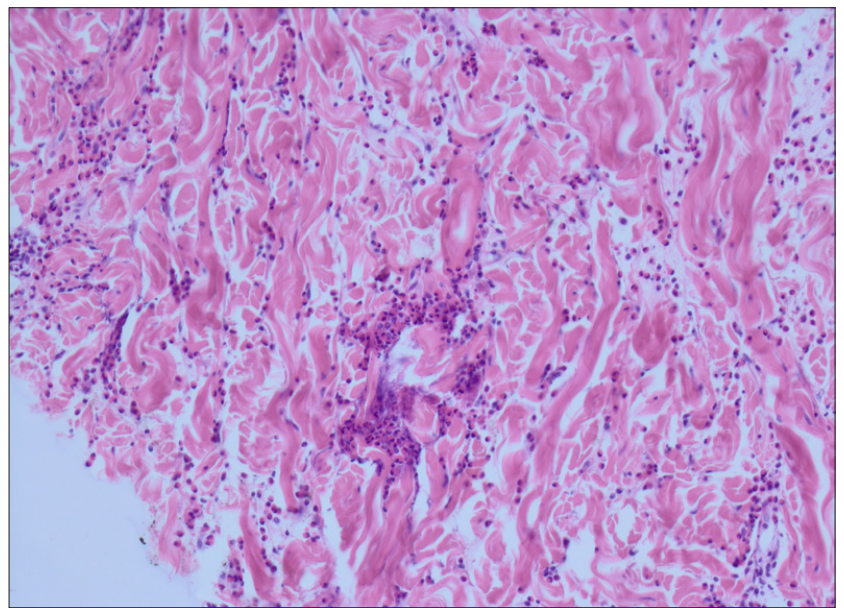

Figure 6. Flame-shaped areas consisting of amorphous deposition of collagen coated with eosinophils

Final diagnosis: Wells Syndrome triggered by insect bites.

Treatment: Treatment was instituted with oral prednisolone $0.5 \mathrm{mg} / \mathrm{kg}$ per day, fexofenadine hydrochloride twice a day and topical clobethasol propionate cream. Cutaneous symptoms improved within a few days, leaving residual erythema and slate-blue hyperpigmentation (Fig. 1). Oral steroid treatment was tapered over eight weeks. Topical treatment was continued with tacrolimus cream. The patient was informed about appropriate protection against insect bites. No further relapses were observed in a 3-month followup examination. 


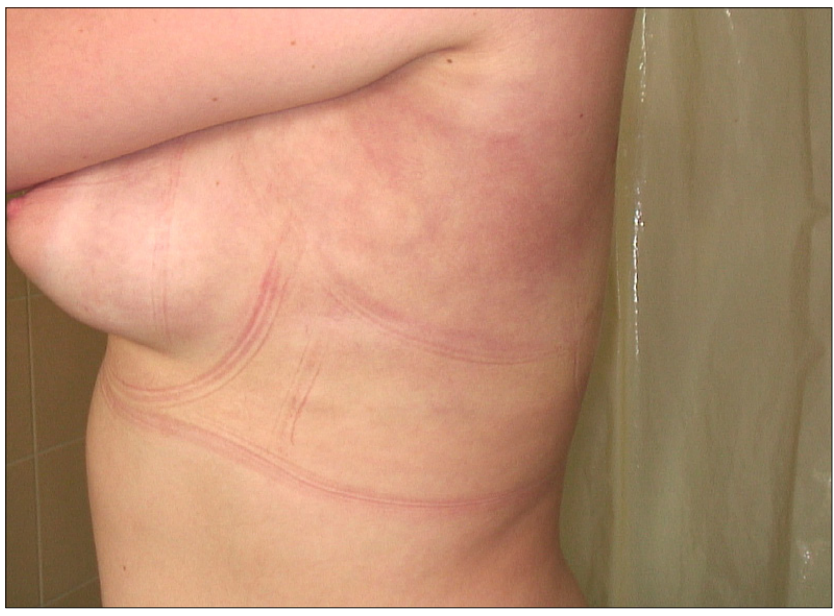

Figure 7. Post-inflammatory residual erythema and slate-blue hyperpigmentation

\section{DISCUSSION}

Eosinophilic cellulitis (Wells syndrome) is a rare inflammatory skin disorder [1]. It was first described in 1971 by Wells as a recurrent granulomatous dermatitis with eosinophilia, and was later named eosinophilic cellulitis [2]. Cutaneous lesions are variable in appearance and may be similar to cellulitis, urticaria, insect bites, morphea or contact dermatitis. Etiology and pathogenesis of eosinophilic cellulitis remains unknown [3]. Hypersensitivity due to different triggers, such as insect bites or stings, drugs, infections, atopic dermatitis and contact dermatitis have been proposed [4]. In several cases, the disease has been found to be associated with myeloproliferative disorders and eosinophilic granulomatosis with polyangitis $[5,6]$. Causative medications include antibiotics, anticholinergic agents, anaesthetics, non-steroidal anti-inflammatory agents, thyroid medications, chemotherapeutic agents, thiomersal containing vaccinations, anti-tumour necrosis factor agents and thiazide diuretics [7]. Activated eosinophils play a major role in the pathogenesis of this dermatosis [4]. Moreover, in patient with eosinophilia it is thought that IL-2 primes eosinophil degranulation $[4,8]$. Wells syndrome usually affects adults, but it has been known to occur in children $[9$, 10]. Most cases are sporadic. The disease is characterized by recurrent episodes of sudden outbreaks of well-circumscribed annular or circinate erythematous-edematous patches that rapidly progress over a few days to morphea-like blue-slatecoloured plaques, with violaceous edges. Papulovesicular, blistering and nodular lesions, which are often painful or pruritic can also be seen $[1,2,11]$. Some patients may complain about fever, arthralgia, lymphadenopathy and malaise [12]. The symptoms can involve different locations, usually the extremities, trunk and face area [1].

Ruggero Caputo et al. [10] presented seven clinical variants of Wells Syndrome (plaque type, annular granuloma-like, urticaria-like, papulovesicular, bullous, papulonodular, fixe drugeruption-like). The most common clinical feature in children is the plaque-type variant, whereas annular granuloma-like lesions were found the most frequently in adults [10]. The lesions change from bright red to pink-brown, green, brown or slate blue [4]. The inflammatory process Usually resolves spontaneously over $2-8$ weeks, without scarring, although scarring alopecia has been observed [9].
Peripherial blood eosinophilia is non-constant (found in 67\% of cases), an increase in eosinophil cation protein (ECP) and IL-5 levels can be detected $[2,13]$.

The patient in this case report presented the coexistence of 2 different clinical pictures of the disease: recurrent erythematous, associated with tense blisters on dorsal aspect of both feet and morphea-like eruptions with violaceous border on the trunk. Cutaneous symptoms improved within a few days of corticosteroid therapy, leaving residual erythema and slate-blue hyperpigmentation. The most common clinical mimics are bacterial cellulitis and erysipelas [4]. The diagnosis of Wells Syndrome should be considered for patients with presumed cellulitis with an atypical presentation, accompanying pruritis, which is often the primary symptom or not responding to appropriate antibiotic [12]. The histopathologic findings in both these soft tissue infections can also reveal oedema, but neutrophils are the predominant inflammatory cells [4]. Other conditions, which should be excluded are alergic contact dermatitis, chronic uriticaria, drug eruptions, arachid bites, insect bites, eosinophilic granulomatosis with polyangitis, eosinophilic fascitis, granuloma annulare, Lyme disease, pediatric hypereosinophilic syndrome, bullous pemphigoid, morphea [13].

Kara Heelan et al. propose diagnostics criteria for Wells Syndrome (Tab. 1) [7]. Three histopathological stages can be distinguished: 1) acute stage with dermal oedema and eosinophilic infiltration of the dermis without signs of vasculitis, 2) sub-acute stage with characteristic brightly flame figures, consisting of amorphous deposition of collagen coated with eosinophils and histiocytes, were seen in about $50 \%$ of the cases. Flame-shaped areas, although distinguished, are nondiagnostic finding, as they can be observed in parasitic infections, follicular mucinosis, Herpes gestationis, eosinophilic granulomatosis with polyangitis, eczema, tinea, scabies, insect bites. 3) Regressive phase is characterized by gradual disappearance of eosinophils with the persistence of histiocytes and apperance of giant cells around collagen deposits, forming microgranulomas $[1,5$, $10,13,14]$. Direct immunofluorescence is negative. In the presented patient, skin biopsy revealed primarily dermal infiltration by eosinophils and flame figures.

There are many therapeutic options for the treatment of eosinophilic cellulitis. The choice of therapy depends on the extent of disease. The medication used for topical therapy are

Table 1. Proposed diagnostic criteria for Wells Syndrome

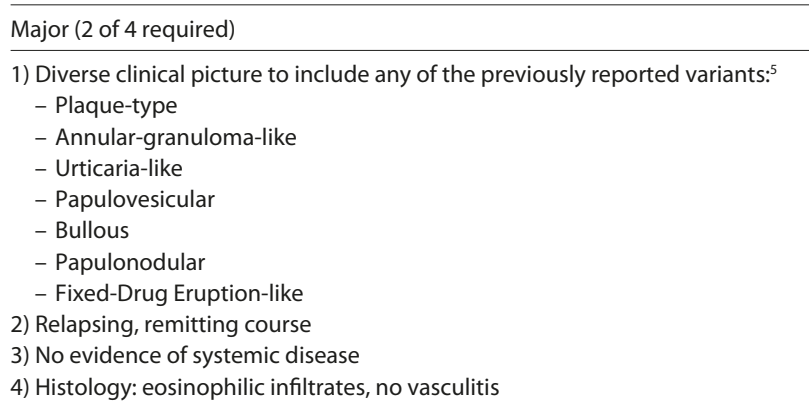

4) Histology: eosinophilic infiltrates, no vasculitis

Minor (at least 1 required)

1) Flame figures

2) Histology: Granulomatous change

3) Peripheral eosinophila, not persistent, not bigger than $>1500 / \mu l$ 4) Triggering factor (e.g. drug) 
glicocorticosteroids and calicineurin inhibitors. Systemic glicocorticosteroids are the most common and effective treatment modality reported so far, but they may lead to corticosteroid dependence with relapses in a short time period [1]. The recommended initial dose for prednisolone is $1-2 \mathrm{mg} / \mathrm{kg}$ per day, continuing with $5 \mathrm{mg} /$ day later on $[1,15$, 16], or starting with $2 \mathrm{mg} / \mathrm{kg}$ for 5-7 days and then tapering the dose [17]. The other therapeutic option is therapy with $1 \mathrm{mg} / \mathrm{kg}$ per day prednisolone $[1,18]$. In the presented case, the lower dose $(0.5 \mathrm{mg} / \mathrm{kg}$ per day) oral prednisolone with antihistamines and topical anti-inflammatory treatment was sufficient to achieve clinical remission. Cyclosporine (1.25$2.5 \mathrm{mg} / \mathrm{kg} /$ day), azatioprine, colchicine, antimalar drugs, dapsone, sulphone/sulfasalazine, minocycline, griseofulvin, PUVA therapy, interferon alpha, interferon gamma and TNF alpha inhibitors have been tried with varying success [1].

Combinations of the above medications are often applied, while response to treatment is not complete. For mild cases topical treatment may be sufficient. The disease may have a recurrent course, with exacerbations and remissions occurring over several years.

\section{CONCLUSION}

Because of the variety of clinical presentations of Wells Syndrome, lack of characteristic features and the selflimiting course, many result in cases being misdiagnosed. The differential diagnosis is also broad. As in the presented case, several biopsy sometimes required to establish proper diagnosis. The lower dose $(0.5 \mathrm{mg} / \mathrm{kg}$ per day) oral prednisolone with antihistamines and topical antiinflammatory treatment, in combination with personal protection from insects bites, can be effective.

In patients with eosinophilic cellulitis triggered by arthropod assault, the prevention of insects is particularly important. The patient should wear fully covering clothing and footwear, and apply insect repellents containing DEET (diethyltoluamide) to exposed skin.

\section{Acknowledgement}

The authors express their thanks dr Janusz Kopczyński for the microscopic photos.

\section{REFERENCES}

1. Räßler F, Lukács J, Elsner P. Treatment of Eosinophilic Cellulitis (Wells Syndrome) - a Systematic Review. J Eur Acad Dermatol Venereol. 2016; 30(9): 1465-79.

2. Ferreli C, Pinna AL, Atzori L, Aste N. Eosinophilic Cellulitis (Wells' Syndrome): A New Case Description. J Eur Acad Dermatol Venereol. 1999; 13(1): 41-45.

3. Fisher GB, Greer KE, Cooper PH. Eosinophilic Cellulitis (Wells' Syndrome). Int J Dermatol. 1985; 24(2): 101-107.

4. Bolognia JL, Schaffer JV, Cerroni L, Dermatology. 4 ed. Elsevier, 2017.

5. Aberer W, Konrad K, Wolff K. Wells' Syndrome Is a Distinctive Disease Entity and Not a Histologic Diagnosis. J Eur Acad Dermatol. 1988; 18 (1Pt 1): 105-14.

6. Schuttelaar M.-La, Jonkman MF. Bullous Eosinophilic Cellulitis (Wells' Syndrome) Associated with Churg-Strauss Syndrome. J Eur Acad Dermatol. 2003; 17(1): 91-93.

7. Heelan K, Ryan JF, Shear NH, Egan CA. Wells syndrome (eosinophilic cellulitis): Proposed diagnostic criteria and a literature review of the drug-induced variant. J Dermatol Case Rep. 2013; 7(4): 113-20.

8. Simon HU, Plötz S, Simon D, Seitzer U, Braathen LR, Menz G, Straumann A, Dummer R, LeviSchaffer F. Interleukin-2 Primes Eosinophil Degranulation in Hypereosinophilia and Wells' Syndrome. Eur J Immunol. 2003; 33(4): 834-39.

9. Nielsen T, Schmidt H, Søgaard H. Eosinophilic Cellulitis. (Well's Syndrome) in a Child. Arch Dermatol. 1981; 117(7): 427-9.

10. Caputo R, Marzano AV, Vezzoli P, Lunardon L. Wells syndrome in adults and children: a report of 19 cases. Arch Dermatol. 2006; 142(9): $1157-61$.

11. Anhi Lee. Eosinophilic Cellulitis (Wells' Syndrome). Korean J Pathol. 1995; 29(3): 407-10.

12. Sinno H, Lacroix JP, Lee J, Izadpanah A, Borsuk R, Watters K, Gilardino M. Diagnosis and Management of Eosinophilic Cellulitis (Wells' Syndrome): A Case Series and Literature Review. Can J Plast Surg. 2012; 20(2): 91-7.

13. Schwartz RA. Wells Syndrome: Background, Pathophysiology, Etiology. Medscape https://emedicine.medscape.com/article/1124844-overview (access 2018).

14. Nacaroglu HT, Celegen M, Karkıner CS, Günay I, Diniz G, Can D. Eosinophilic cellulitis (Wells' syndrome) caused by a temporary henna tattoo Postępy Dermatol Alergol. 2014; 31(5): 322-4 31.

15. Goh CL. Eosinophilic Cellulitis (Wells'syndrome). Int J Dermatol. 1992; 31(6): 429-30

16. Tassava T, Rusonis PA, Whitmore SE. Recurrent Vesiculobullous Plaques. Eosinophilic Cellulitis (Wells Syndrome). Arch Dermatol. 1997; 133(12): 1580-1, 1583-4.

17. Gilliam AE, Bruckner AL, Howard RM, Lee BP, Wu S, Frieden IJ. Bullous "cellulitis" with eosinophilia: case report and review of Wells' syndrome in childhood. Pediatrics. 2005; 116(1): e14955.

18. Coldiron BM, Robinson JK. Low-Dose Alternate-Day Prednisone for Persistent Wells' Syndrome. Arch Dermatol. 1989; 125(12): 1625-6. 